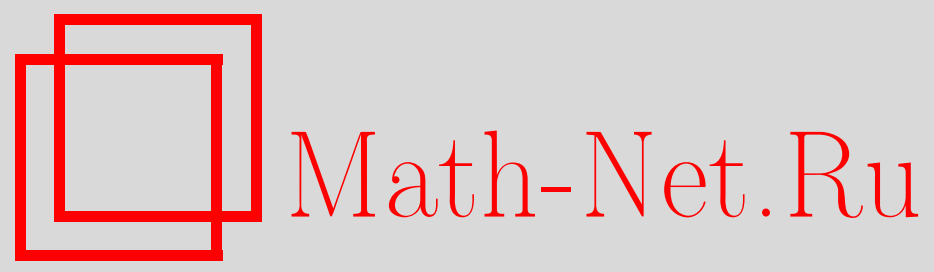

А. Б. Мухин, Оценки локальных вероятностей сверток дискретных распределений, Дискрет. матем., 1999, том 11, выпуск 2, 103-111

DOI: https://doi.org/10.4213/dm375

Использование Общероссийского математического портала Math-Net.Ru подразумевает, что вы прочитали и согласны с пользовательским соглашением http://www . mathnet.ru/rus/agreement

Параметры загрузки:

IP : 3.80 .181 .102

26 апреля 2023 г., 04:38:14

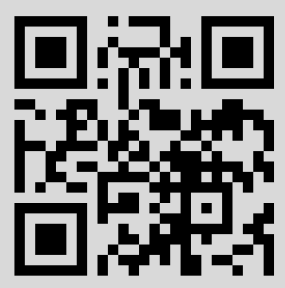




\title{
Оценки локальных вероятностей сверток дискретных распределений
}

\author{
(c) 1999 г. А. Б. Мухин
}

\begin{abstract}
Для сумм независимых одинаково распределенных случайных величин с конечным числом возможных значений изучается асимптотическое поведение максимума вероятностей отдельных значений с ростом числа слагаемых. Рассматриваются малоизученные случаи, когда распределение слагаемых нерешетчато или зависит от числа слагаемых (схема серий).
\end{abstract}

Пусть $G(s)$ - класс распределений $F$ вещественных случайных величин (с. в.) с $s$ значениями;

$$
P_{n}(F, m)=F^{* n}(m)=\mathbf{P}\left\{S_{n}=m\right\}
$$

где

$$
S_{n}=\sum_{k=1}^{n} X_{k},
$$

$X_{k}$ - независимые для $k=1, \ldots, n$ с. в. с распределением $F$. Работа посвящена оценкам величин

$$
P_{n}(F)=\sup _{m \in \mathbf{R}} P_{n}(F, m)
$$

Хорошо известны общие неравенства для функций концентрации сумм независимых с. в. и $P_{n}(F)$ (см. [1-3] и др.). Однако, эти неравенства при $F \in G(s)$ дают порядок убывания $O\left(n^{-1 / 2}\right)$, в том числе для $F$, зависящих от $n$. Это, конечно, далеко от экстремального порядка убывания, как будет видно из дальнейшего. Б. А. Рогозиным [4] получены точные неравенства для $P_{n}(F)$ с помощью сверток соответствующих равновероятных распределений. Легко заметить, что и с помощью этих неравенств для $F \in G(s)$ можно получить лишь оценки порядка $O\left(n^{-1 / 2}\right)$. Некоторые результаты с более высоким, чем $n^{-1 / 2}$, порядком убывания имеются в $[5,6]$.

Пусть $a_{i}$ - возможные значения с. в. $X_{k}$ с распределением $F \in G(s)$ и

$$
p_{i}=F\left(a_{i}\right)=\mathbf{P}\left\{X_{k}=a_{i}\right\}, \quad i=1, \ldots, s .
$$

Будем считать (это, очевидно, не ограничивает общности постановки задачи), что

$$
a_{1}=0, \quad p_{1}=\max _{1 \leqslant k \leqslant n} p_{k}, \quad\left|a_{i+1}\right| \geqslant\left|a_{i}\right|
$$


Очень просто решается вопрос об экстремальном порядке убывания $P_{n}(F)$. Положим

$$
P_{n s}=\inf _{F \in G(s)} P_{n}(F) .
$$

Теорема 1. Для любого $s \in \mathbf{N}$ при $n \rightarrow \infty$ справедлива асимптотическая формула

$$
P_{n s}=\sqrt{2 \pi}(2 \pi s)^{-s / 2} n^{-(s-1) / 2}+o\left(n^{-(s-1) / 2}\right) .
$$

Доказательство. Выражая $P_{n}(F, m)$ с помощью полиномиальных вероятностей

$$
P_{n}\left(m_{1}, \ldots, m_{s} ; p_{1}, \ldots, p_{s}\right)=n ! \prod_{i=1}^{s} \frac{1}{m_{!}} p_{i}^{m_{i}}
$$

и считая $0^{0}=1$, получим, что

$$
\begin{aligned}
P_{n s} & =\inf _{F \in G(s)} \sup _{m \in \mathbf{R}} \sum_{a_{1} m_{i}+\ldots+a_{s} m_{s}=m} P_{n}\left(m_{1}, \ldots, m_{s} ; p_{1}, \ldots, p_{s}\right) \\
& \geqslant \inf _{p_{1}+\ldots+p_{s}=1} \sup _{m_{1}+\ldots+m_{s}=n} P_{n}\left(m_{1}, \ldots, m_{s} ; p_{1}, \ldots, p_{s}\right) .
\end{aligned}
$$

С другой стороны, как легко заметить, для $F$ таких, что $a_{1}=0, a_{2}=1, a_{3}>$ $n, \ldots, a_{s}>n a_{s-1}$

$$
P_{n}\left(F^{\prime}\right)=\sup _{m_{1}+\ldots+m_{s}=n} P_{n}\left(m_{1}, \ldots, m_{s} ; p_{1}, \ldots, p_{s}\right) .
$$

Следовательно, для любых $n \in \mathbf{N}, s \in \mathbf{N}$

$$
P_{n s}=\inf _{p_{1}+\ldots+p_{s}=1} \sup _{m_{1}+\ldots+m_{s}=n} P_{n}\left(m_{1}, \ldots, m_{s} ; p_{1}, \ldots, p_{s}\right) .
$$

Пусть

$$
P_{n}(F)=P_{n}\left(\bar{m}_{1}, \ldots, \bar{m}_{s} ; p_{1}, \ldots, p_{s}\right),
$$

то есть $\bar{m}_{i}=\bar{m}_{i}\left(n, p_{1}, \ldots, p_{s}\right)$ - точки, где достигается супремум.

Используя формулу

$$
\frac{P_{n}\left(m_{1}, \ldots, m_{i}, \ldots, m_{j}, \ldots, m_{s} ; p_{1}, \ldots, p_{s}\right)}{P_{n}\left(m_{1}, \ldots, m_{i}-1, \ldots, m_{j}+1, \ldots, m_{s} ; p_{1}, \ldots, p_{s}\right)}=\frac{p_{i}\left(m_{j}+1\right)}{p_{j} m_{i}}
$$

легко показать, что $\bar{m}_{i}$ отличаются от $n p_{i}$ не более чем на $s$. Следовательно, для любых $p_{i} \in(0,1)$

$$
\left|\bar{m}_{i}-n p_{i}\right|=O\left(\sqrt{n p_{i}\left(1-p_{i}\right)}\right)
$$

и эти точки находятся в зоне действия локальной предельной теоремы для полиномиальной схемы. Поэтому

$$
\begin{aligned}
P_{n}(F) & =\sup _{m_{1}+\ldots-m_{s}} \frac{(2 \pi n)^{-(s-1) / 2}}{\sqrt{p_{1} \ldots p_{s}}} \exp \left\{-\sum_{i=1}^{s} \frac{\left(m_{i}-n p_{i}\right)^{2}}{2 n p_{i}}\right\}(1+o(1)) \\
& =\frac{(2 \pi n)^{-(s-1) / 2}}{\sqrt{p_{1}} \frac{p_{s}}{\ldots+o(1)) .}} .
\end{aligned}
$$

Отсюда, учитывая непрерывность $P_{n}(F)$ как функции от $p_{i}$, легко получаем утверждение теоремы. 
Переходя к общим оценкам для $P_{n}(F)$, положим

$$
\begin{gathered}
B_{n}^{2}=n \mathbf{D} X_{1}, \quad M_{n}^{2}=n \mathbf{M} X_{1}^{2}, \\
M_{n}(u)=n \sum_{i:\left|a_{i}\right| \leqslant u} a_{i}^{2} p_{i} .
\end{gathered}
$$

Мы сохраняем эти обозначения и в случае, когда распределение $F$ (то есть $a_{i}$ и $p_{i}$, $i=1, \ldots, s)$ зависит от $n$. Введем также обозначение

$$
B_{n}^{2}(u)=n \int_{|x| \leqslant u} x^{2} F^{*}(d x)
$$

где $F^{*}$ - распределение симметризованных слагаемых $X_{k}^{*}, k=1, \ldots, n$. При предположениях (1) относительно $F$

$$
\mathbf{P}\left\{X_{k}^{*}=a_{i}\right\} \geqslant p_{i} p_{1} \geqslant \frac{p_{i}}{s}
$$

поэтому

$$
B_{n}^{2}(u) \geqslant \frac{1}{s} M_{n}^{2}(u), \quad B_{n}^{2} \geqslant \frac{1}{2 s} M_{n}^{2}
$$

Отметим также, что $B_{n}^{2} \leqslant M_{n}^{2}$.

Теорема 2. Пусть $u_{0}, u_{1}, \ldots, u_{r}$ - произволъный набор положителънъх чисел. Тогда при $r \geqslant 1$

$$
P_{n}(F) \leqslant \frac{(c \sqrt{s})^{r+1} u_{0}}{M_{n}\left(u_{r}\right)} \prod_{i=1}^{r}\left(1+\frac{u_{i}}{M_{n}\left(u_{i-1}\right)}\right)
$$

npu $r=0$

$$
P_{n}(F) \leqslant \frac{c u_{0} \sqrt{s}}{M_{n}\left(u_{0}\right)}
$$

здесь и далее с - положителъные абсолютные постоянные, вообще говоря, различнаме.

Неравенство (5) аналогично неравенствам из теоремы 3.2 в [2] или теоремы 2 из [5]. Прежде чем давать доказательство теоремы 2, приведем некоторые следствия, иллюстрирующие схему использования неравенства (4).

Следствие 1. Если при каких либо $\alpha_{i} \in(0,1]$

$$
\left|a_{i+1}\right| \geqslant \alpha_{i+1} M_{n}\left(a_{i} / \alpha_{i}\right)
$$

$\partial \Omega \Omega i=2, \ldots, s-1, m o$

$$
P_{n}(F) \leqslant \frac{(c \sqrt{s})^{s-1}}{\prod_{i=2}^{s} \alpha_{i} \sqrt{p}_{i}} n^{-(s-1) / 2} .
$$


Доказательство. Положим в теореме 2

$$
u_{i}=\left|a_{i+2}\right| / \alpha_{i+2}, \quad i=0, \ldots, s-2, \quad r=s-2,
$$

тогда $u_{i+1} \geqslant M_{n}\left(u_{i}\right)$ и

$$
P_{n}(F) \leqslant \frac{(c \sqrt{s})^{s-1} 2^{s-2} \prod_{i=0}^{s-2} u_{i}}{\prod_{i=1}^{s-1} M_{n}\left(u_{i-1}\right)} \leqslant \frac{(c \sqrt{s})^{s-1} \prod_{i=0}^{s-2} u_{i}}{\prod_{i=1}^{s-1} M_{n}\left(a_{i+1}\right)} \leqslant \frac{(c \sqrt{s})^{s-1} \prod_{i=0}^{s-2} a_{i+2} / \alpha_{i+2}}{\prod_{i=1}^{s-1} a_{i+1} \sqrt{n p_{i+1}}}
$$

откуда следует (6).

Следствие 2. Для любого $u_{0}>0$ и натурального $r$ справедливы неравенства

$$
P_{n}(F) \leqslant \frac{(c \sqrt{s})^{r+1} u_{0}}{M_{n}\left(M_{n} \ldots M_{n}\left(u_{0}\right) \ldots\right)}
$$

где в знаменателе стоит $r$-кратная итерация бункции $M_{n}(u)$.

Неравенство (7) следует из (4), если положить $u_{i}=M_{n}\left(u_{i-1}\right)$ для $i=1, \ldots, r$. Полагая $u_{i}=\beta_{i} M_{n}\left(u_{i-1}\right)$ с $\beta_{i}>0$, из (4) получаем неравенство

$$
P_{n}(F) \leqslant \frac{(c \sqrt{s})^{r+1} u_{0} \prod_{i=1}^{r}\left(1+\beta_{i}\right)}{M_{n}\left(\beta_{r} M_{n}\left(\ldots \beta_{1} M_{n}\left(u_{0}\right) \ldots\right)\right)},
$$

которое может оказаться более полезным, чем (7). Если, например,

$$
a_{1}=0, \quad a_{2}=1, \quad a_{3}=n^{\alpha}, \quad p_{1}=p_{2}=p_{3}=1 / 3,
$$

то из (8) с

$$
r=u_{0}=1, \quad \beta_{1}=\sqrt{3} n^{\alpha-1 / 2}
$$

получим, что

$$
P_{n}(F) \leqslant \begin{cases}c n^{-1} & \text { при } \alpha \geqslant 1 / 2, \\ c n^{-\alpha-1 / 2} & \text { при } 0<\alpha \leqslant 1 / 2 .\end{cases}
$$

Из (5) и (7) при $\alpha \geqslant 1 / 2$ можно получить лишь оценку $P_{n}(F)=O(1 / \sqrt{n})$.

Доказательство теоремъ 2. Будем обозначать $f(X, t)$ характеристическую функцию $X$ и $f_{n}(t)=f\left(S_{n}, t\right)$. В силу известной формулы обращения (см. например теорему 3.2 .3 в [7])

$$
P_{n}(F) \leqslant \frac{1}{2} \lim _{T \rightarrow \infty} \frac{1}{T} \int_{|t| \leqslant T}\left|f_{n}(t)\right| d t .
$$

Разбивая область интегрирования и считая $T$ кратным $1 / u_{0}$, получаем неравенство

$$
P_{n}(F) \leqslant u_{0} \sup _{D \in R} \int_{D}^{D+1 / u_{0}}\left|f_{n}(t)\right| d t .
$$


Вновь разбиваем область интегрирования и используем теорему о среднем:

$$
\begin{aligned}
P_{n}(F) & \leqslant u_{0} \sup _{D} \sum_{r=0}^{\left[u_{1} / u\right]} \int_{D+r / u_{1}}^{D+(r+1) / u_{1}}\left|f_{n}(t)\right| d t \\
& \leqslant u_{0}\left(\sup _{D} \int_{D}^{D+1 / u_{1}}\left|f_{n}(t)\right|^{1 / 2} d t\right) \sup \sum_{r=0}^{\left[u_{1} / u_{0}\right]}\left|f_{n}\left(t_{r}\right)\right|^{1 / 2}
\end{aligned}
$$

где второй супремум берется по всем $D \in \mathbf{R}$ и $t_{r} \in\left[D+r / u_{1}, D+(r+1) / u_{1}\right]$, причем можно считать, что все $t_{r} \in\left[D, D+1 / u_{0}\right]$.

Далее существенную роль будут играть оценки характеристических функций сумм в среднем. Положим

$$
\begin{gathered}
I_{n}(\Delta, \beta)=\sup _{D} \int_{D}^{D+\Delta}\left|f_{n}(t)\right|^{\beta} d t, \\
\Sigma_{n}(\Delta, \delta, \beta)=\sup _{D} \sup _{\left\{t_{r}\right\}} \sum_{r=0}^{[\Delta / \delta]}\left|f_{n}\left(t_{r}\right)\right|^{\beta} .
\end{gathered}
$$

где верхняя грань берется по $D \in \mathbf{R}$ и всем $t_{r} \in[D+r \delta, D+(r+1) \delta]$, причем $t_{r} \in[D, D+\Delta]$.

В [8] доказана оценка

$$
I_{n}(\Delta, \beta) \leqslant \frac{3 \pi \sqrt{\pi}}{\sqrt{2 \beta} B_{n}(1 / \Delta)} .
$$

Аналогичным путем оценим $\Sigma_{n}(\Delta, \delta, \beta)$. Очевидно, что

$$
\begin{aligned}
\left|f_{n}(t)\right|=\left|f\left(X_{1}, t\right)\right|^{n} & \leqslant \exp \left\{-\frac{n}{2}\left(1-\left|f\left(X_{1}, t\right)\right|^{2}\right)\right\} \\
& =\exp \left\{-\frac{n}{2} \int(1-\cos t x) F^{*}(d x)\right\} \\
& \leqslant \exp \left\{-n \int_{|x| \leqslant 1 / \Delta} \sin ^{2} \frac{t x}{2} F^{*}(d x)\right\} .
\end{aligned}
$$

Для $t_{r}=D+\left(r+\theta_{r}\right) \delta \in[D, D+\Delta]$, где $\theta_{r} \in[0,1]$, учитывая периодичность функции $\sin ^{2} z$, находим, что

$$
\begin{aligned}
h\left(t_{r}, \Delta\right) & =\int_{|x| \leqslant 1 / \Delta} \sin ^{2} \frac{t_{r} x}{2} F^{*}(d x)=\int_{|x| \leqslant 1 / \Delta} \sin ^{2}\left(D x / 2+\left(r+\theta_{r}\right) \delta x / 2\right) F^{*}(d x) \\
& =\int_{|x| \leqslant 1 / \Delta} \sin ^{2}\left(v+\left(r+\theta_{r}\right) \delta x / 2\right) F^{*}(d x),
\end{aligned}
$$


где $v=v(x, D)$, но $|v| \leqslant \pi / 2$ и $v$ не зависит от $r$. Для $0<\left(r+\theta_{r}\right) \delta \leqslant \Delta$ и $|x| \leqslant 1 / \Delta$

$$
\left|v+\left(r+\theta_{r}\right) \delta x / 2\right| \leqslant \frac{\pi+1}{2},
$$

поэтому

$$
\begin{aligned}
h\left(t_{r}, \Delta\right) \geqslant c & \int_{|x| \leqslant 1 / \Delta}\left(v+\frac{\left(r+\theta_{r}\right) \delta x}{2}\right)^{2} F^{*}(d x) \\
= & c\left(B_{1}^{2}(1 / \Delta)\left(\frac{\left(r+\theta_{r}\right) \delta}{2}+B_{1}^{-2}(1 / \Delta) \int_{|x| \leqslant 1 / \Delta} v x F^{*}(d x)\right)^{2}\right. \\
& \left.+\int_{|x| \leqslant 1 / \Delta} v^{2} F^{*}(d x)-B_{1}^{-2}(1 / \Delta)\left(\int_{|x| \leqslant 1 / \Delta} v x F^{*}(d x)\right)^{2}\right) .
\end{aligned}
$$

Применяя неравенство Коши к

$$
\int_{|x| \leqslant 1 / \Delta} v x F^{*}(d x)
$$

получим, что

$$
h\left(t_{r}, \Delta\right) \geqslant c B_{1}^{2}(1 / \Delta)\left(\left(r+\theta_{r}\right) \delta / 2+A\right)^{2}
$$

где

$$
A=B_{1}^{-2}(1 / \Delta) \int_{|x| \leqslant 1 / \Delta} v x F^{*}(d x)=A(F, D, \Delta),
$$

но $A$ не зависит от $r$. Из (11) и последней оценки следует, что

$$
\Sigma_{n}(\Delta, \delta, \beta) \leqslant \sup _{D} \sup _{\left\{t_{r}\right\}} \sum_{r=0}^{[\Delta / \delta]} \exp \left\{-c \beta B_{n}^{2}(1 / \Delta)\left(\left(r+\theta_{r}\right) \delta / 2+A\right)^{2}\right\} .
$$

Используя свойства функции $\exp \left\{-c x^{2}\right\}$, находим, что

$$
\begin{aligned}
\Sigma_{n}(\Delta, \delta, \beta) & \leqslant 2 \sum_{j=0}^{\infty} \exp \left\{-c \beta B_{n}^{2}(1 / \Delta) j^{2} \delta^{2}\right\} \\
& \leqslant 2+2 \int_{0}^{\infty} \exp \left\{-c \beta \delta^{2} B_{n}^{2}(1 / \Delta) x^{2}\right\} d x \\
& \leqslant 2+\frac{c}{\sqrt{\beta} \delta B_{n}(1 / \Delta)} .
\end{aligned}
$$

Таким образом, для любых $\beta>0, \Delta>0, \delta>0$

$$
\Sigma_{n}(\Delta, \delta, \beta) \leqslant c\left(1+\frac{1}{\sqrt{\beta} \delta B_{n}(1 / \Delta)}\right) .
$$


Из (9), (12) и (3) получаем оценки

$$
\begin{aligned}
P_{n}(F) & \leqslant u_{0} I_{n}\left(1 / u_{1}, 1 / 2\right) \sum_{n}\left(1 / u_{0}, 1 / u_{1}, 1 / 2\right) \\
& \leqslant c u_{0} I_{n}\left(1 / u_{1}, 1 / 2\right)\left(1+\sqrt{2} u_{1} / B_{n}\left(u_{0}\right)\right) \\
& \leqslant c \sqrt{s} u_{0} I_{n}\left(1 / u_{1}, 1 / 2\right)\left(1+u_{1} / M_{n}\left(u_{0}\right)\right) .
\end{aligned}
$$

Аналогичным образом, разбивая область интегрирования в $I_{n}\left(1 / u_{1}, 1 / 2\right)$ и повторяя эту процедуру $r$ раз, получим, что

$$
P_{n}(F) \leqslant(c \sqrt{s})^{r} u_{0} I_{n}\left(1 / u_{r}, 1 / 2^{r}\right) \prod_{i=1}^{r}\left(1+u_{i} / M_{n}\left(u_{i-1}\right)\right)
$$

Для доказательства (4) осталось использовать (10) и (3). Доказательство (5) очевидно.

Для фиксированных (не зависящих от $n$ ) решетчатых распределений $F$ асимптотическое поведение $P_{n}(F, m)$ и $P_{n}(F)$ хорошо изучено (см., например, [9] и имеющуюся там библиографию). Рассмотрим случай фиксированных не обязательно решетчатых $F \in G(s)$.

Теорема 3. Если среди $a_{2}, a_{3}, \ldots, a_{s}$ имеется $\nu$ значений $a_{i_{1}}, a_{i_{2}}, \ldots, a_{i_{\nu}}$ таких, ymo

$$
\sum_{j=1}^{\nu} m_{j} a_{i_{j}} \neq 0
$$

для любых целых $m_{j}$ не равных нулю одновременно (то есть $a_{i}$ являются линейно независимыми элементами векторного пространства размерности, не менъшей $\nu$, над полем цельх чисел), то

$$
P_{n}(F)=O\left(n^{-\nu / 2}\right)
$$

В данном случае можно описать поведение $P_{n}(F)$ и $P_{n}(F, m)$ более точно. Теорема 3 легко получается из приводимого ниже утверждения.

Пусть для $i=2, \ldots, s$

$$
a_{i}=\sum_{j=1}^{\nu} r_{i j} h_{j}
$$

где $h_{j}$ - некоторые вещественные числа, $h_{j} \neq 0, r_{i j} \in \mathbf{Z}, \nu=1, \ldots, s-1$, причем $\nu-$ минимальное число, при котором существует такое представление ( $\nu=1$ соответствует случаю решетчатых распределений). Рассмотрим $\nu$-мерный случайный вектор $\bar{X}$ с целочисленными компонентами такой, что $\mathbf{P}\left\{\bar{X}=\bar{r}_{i}\right\}=p_{i}$, где $\bar{r}_{i}=\left(r_{i 1}, \ldots, r_{i \nu}\right)$ для $i \geqslant 2$ и $\bar{r}_{1}=(0, \ldots, 0)$. Пусть $Q-$ матрица ковариаций вектора $\bar{X}$ и

$$
\Omega=\left\{x=\left(x_{1}, \ldots, x_{\nu}\right):|x|_{i} \leqslant 1 / 2,|x|>0\right\}
$$

- $\nu$-мерный куб без центральной точки. 
Теорема 4. Если для любого $d \in \Omega$ существует $i=2, \ldots, s$ такое, что $\left(\bar{r}_{i}, d\right) \in \mathbf{Z}$, mo $\partial \Omega \Omega$

$$
m=\sum_{j=1}^{\nu} m_{j} h_{j}, \quad m_{j} \in Z
$$

справедлива оценка

$$
P_{n}\left(F, m_{i}\right)=n^{-\nu / 2} \varphi_{Q}\left(\frac{\bar{m}-n \mathbf{M} \bar{X}}{\sqrt{n}}\right)+o\left(n^{-\nu / 2}\right),
$$

где $\bar{m}=\left(m_{1}, \ldots, m_{\nu}\right), \varphi_{Q}-$ плотность $\nu$-мерного нормалъного закона с нулевъм средним и матрицей ковариачий $Q$. Соотношение (14) выполняется равномерно по всем $m$ с челыми компонентами. Отсюда,

$$
P_{n}(F)=(2 \pi n)^{-\nu / 2}(\operatorname{det} Q)^{-1 / 2}+o\left(n^{-\nu / 2}\right) .
$$

При $\nu=1$ условие теоремы эквивалентно требованию, чтобы $h_{1}$ было максимальным шагом решетчатых случайных величин $X_{k}$, так что в этом случае получаем классическую локальную предельную теорему для решетчатых случайных величин.

Доказательство. В силу минимальности $\nu$ в выражении (13) представление

$$
m=\sum_{j=1}^{\nu} m_{j} h_{j}
$$

как легко проверить, является единственным, то есть из

$$
\sum_{j=1}^{\nu} m_{j} h_{j}=\sum_{j=1}^{\nu} m_{j}^{\prime} h_{j}
$$

следует, что $m_{j}=m_{j}^{\prime}$ для любого $j=1, \ldots, \nu$. Поэтому

$$
P_{n}(F, m)=\mathbf{P}\left\{S_{n}=\sum_{j=1}^{\nu} m_{j} h_{j}\right\}=\mathbf{P}\left\{\sum_{k=1}^{n} \bar{X}_{k}=\bar{m}\right\},
$$

где $\bar{X}_{k}$ - независимые случайные $\nu$-мерные векторы с тем же распределением, что у $\bar{X}$. Поскольку распределение

$$
n^{-1 / 2}\left(\sum_{k=1}^{n} \bar{X}_{k}-n \mathbf{M} \bar{X}_{1}\right)
$$

слабо сходится к нормальному с нулевым средним и матрицей ковариаций $Q$, доказательство (14) сводится к проверке справедливости локальной предельной теоремы для $\left\{\bar{X}_{k}\right\}$. Из теоремы 1 работы [9] следует, что локальная предельная теорема в случае одинаково распределенных случайных величин (в том числе и многомерных) эквивалентна выполнению интегральной предельной теоремы и условия

$$
H\left(\bar{X}_{1}, d\right)=M\left\langle\left(\bar{X}_{1}^{*}, d\right)\right\rangle^{2}>0
$$


для $d \in \Omega$, где $\bar{X}_{1}^{*}-$ симметризованная случайная величина $\bar{X}_{1},\langle\cdot\rangle-$ расстояние до ближайшего целого числа. Последнее условие эквивалентно в свою очередь равенству единице максимального шага распределения.

В наших предположениях (15) означает, что

$$
\sum_{i, j} p_{i} p_{j}\left\langle\left(\left(\bar{r}_{i}-\bar{r}_{j}\right), d\right)\right\rangle^{2}>0
$$

то есть для любого $d \in \Omega$ существуют $i$ и $j, i \neq j$, такие, что $\left(\left(\bar{r}_{i}-\bar{r}_{j}\right), d\right)$ не целое, что эквивалентно условию теоремы.

Замечание. Если

$$
\sum_{j=2}^{s} l_{j} a_{j} \neq 0
$$

для любых целых $l_{j}$, не равных нулю одновременно, то с помощью элементарной локальной предельной теоремы для последовательности испытаний легко получить более простое представление $P_{n}(F, m)$, чем (14), а именно,

$$
P_{n}\left(F, \sum_{j=2}^{s} m_{j} a_{j}\right)=\frac{(2 \pi n)^{-(s-1) / 2}}{\sqrt{p_{1} \ldots p_{s}}} \exp \left\{-\sum_{j=1}^{s} \frac{\left(m_{j}-n p_{j}\right)^{2}}{2 n p_{j}}\right\}+o\left(n^{-(s-1) / 2}\right)
$$

равномерно по всем целым $m_{j} \geqslant 0$ таким, что $m_{1}+\ldots+m_{s}=n$.

\section{Список литературы}

1. Хенгартнер В., Теодореску Р. Функции концентрации. Наука, Москва, 1980.

2. Esseen C. G. On the concentration function of a sum of independent random variables. $Z$. Wahrsh. Verw Gebiete (1968) 9, 290-308.

3. Ушаков Н. Г. Верхние оценки максимальной вероятности для сумм независимых случайных векторов. Теория вероятностей и ее применения (1985) 30, №1, 33-43.

4. Рогозин Б. А. Неравенства для функций концентрации сверток арифметических распределений и распределений с ограниченными плотностями. Теория вероятностей $u$ ее применения (1987) 32, №2, 351-355.

5. Мухин А. Б. Оченки концентрачии распределений сумм независимых случайных величин. Изд-во Университет, Ташкент, 1995.

6. Мухин А. Б. О концентрации распределений сумм независимых случайных величин. I, II. Известия $А Н$ УзССР, серия физ-матем. н. (1973) №2, 25-29; №6, 18-23.

7. Лукач Е. Характеристические функции. Наука, Москва, 1979.

8. Мухин А. Б. О предельной теореме для плотностей и оценке скорости сходимости. Известия АН УзССР, серия физ-матем. ж. (1971) №5, 13-19.

9. Мухин А. Б. Локальные предельные теоремы для решетчатых случайных величин. Теория вероятностей и ее применения (1991) 36, №4, 660-674.

Статья поступила 12.09.1997.

Переработанный вариант поступил 25.06.1998. 